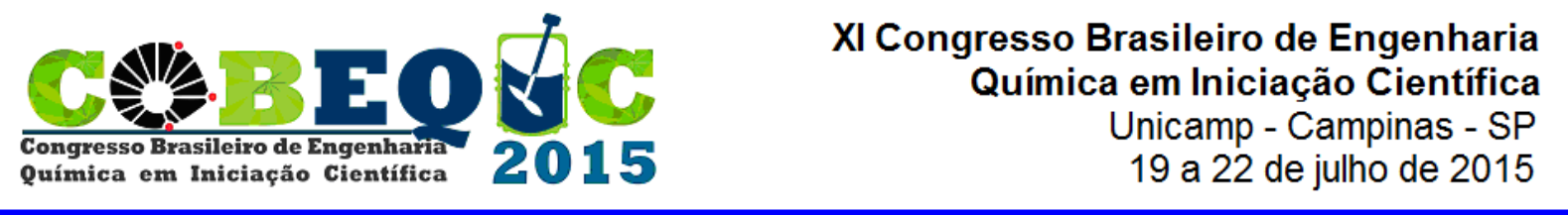

\title{
ESTUDO DA ADSORÇÃO DE CHUMBO UTILIZANDO COMO ADSORVENTE BAGAÇO DE CANA-DE-AÇÚCAR ATIVADO
}

\author{
J. E. M. PAZ ${ }^{1}$, A. C. S. SANTOS ${ }^{1}$, L. M. L. B. ESPÍNOLA ${ }^{1}$, \\ F. D. S. CURBELO ${ }^{1}$, A. I. C. GARNICA ${ }^{1}$ \\ ${ }^{1}$ Universidade Federal da Paraíba, Departamento de Engenharia Química \\ E-mail para contato: eduardomatos3@hotmail.com
}

\begin{abstract}
RESUMO - O adsorvente, bagaço de cana-de-açúcar, foi tratado quimicamente com ácido sulfúrico $\left(1,0 \mathrm{molL}^{-1}\right)$. As soluções sintéticas de efluente foram feitas a partir do cloreto de chumbo $\left(\mathrm{PbCl}_{2}\right)$, obtendo concentrações entre 1 e $50 \mathrm{mg} / \mathrm{L}$ de chumbo. As isotermas de adsorção foram obtidas pelo método do banho finito baseado no balanço de massa do adsorbato. Os ensaios de adsorção foram feitos nas temperaturas de 30,50 e $65^{\circ} \mathrm{C}$ com tempo de equilíbrio de 6 horas, a solução remanescente foi analisada por técnica de espectrofotometria. $\mathrm{O}$ modelo de Langmuir é que melhor descreve o processo, o sistema apresentado é exotérmico e espontâneo e os resultados também mostram que o processo proposto apresenta potencial para o tratamento de efluentes industriais que contenham chumbo.
\end{abstract}

\section{INTRODUÇÃO}

Os despejos de resíduos industriais são as principais fontes de contaminação das águas dos rios com metais pesados, como o chumbo. Esses elementos quando lançados como resíduos industriais, na água, no solo ou no ar, podem ser absorvidos pelos vegetais e animais das proximidades, provocando graves intoxicações ao longo da cadeia alimentar, e por não possuir nenhuma função dentro dos organismos sua acumulação pode provocar graves doenças, sobretudo nos mamíferos, como câncer e outras doenças graves.

O bagaço de cana-de-açúcar é um resíduo da agroindústria sucroalcooleira, sendo o Brasil o maior produtor mundial de cana-de-açúcar. Atualmente a cana-de-açúcar é plantada em vários estados brasileiros, alcançando 648,1 milhões de toneladas no ano de 2013. Do seu processo de industrialização, obtêm-se como produtos açúcar nas suas mais variadas formas e tipos de álcool etílico (anidro e hidratado) (Balanço Energético Nacional, 2014). Entretanto a cada ano são gerados de 5 a 12 milhões de tonelada desse material que corresponde aproximadamente a $30 \%$ da cana moída (Souza et al., 2009). O maior problema que esse setor encontra é o destino final desse material, que, em grande quantidade não possui local específico para ser descartado, o que leva diversos países, inclusive o Brasil, a realizarem pesquisas sobre a utilização desse resíduo como matéria-prima alternativa nos diversos setores industriais, além de preservar o meio ambiente (Souza et. al., 2009). 
Considerando o que foi exposto acima, este trabalho teve como objetivo fundamental o estudo da adsorção do chumbo, a diferentes temperaturas, utilizando o bagaço de cana-deaçúcar como adsorvente.

\section{METODOLOGIA}

Inicialmente o bagaço proveniente da cana-de-açúcar foi lavado abundantemente com água para que materiais indesejáveis fossem removidos, como pedaço de palha, areia ou madeira, que pudessem causar interferências nos resultados experimentais.

Logo após a etapa de lavagem, o bagaço foi seco em estufa a $60{ }^{\circ} \mathrm{C}$, por aproximadamente 40 horas. Depois de seco, o bagaço foi moído em um moinho de facas. Depois de moído, o bagaço foi peneirado utilizando as peneiras da série Tyler de malhas, com o objetivo de obter partículas com diâmetro médio variando entre 0,5 e 2,0 mm.

Em seguida o bagaço da cana-de-açúcar foi submetido a um tratamento químico com ácido sulfúrico $\left(1,0 \mathrm{molL}^{-1}\right)$ com finalidade de modificá-lo e melhorar suas propriedades como adsorvente. Nesse processo o bagaço foi colocado na solução de ácido sulfúrico e deixado em repouso por 24 horas. A proporção sólido-líquido utilizada foi de $0,05 \mathrm{gmL}^{-1}$. Em seguida foi feita a filtração do bagaço para a remoção do ácido, e logo após o bagaço foi lavado com água destilada até o pH estabilizar e ficar em torno de 6.

Para realizar o estudo de adsorção foram preparadas soluções sintéticas de efluente contendo chumbo, as soluções foram feitas a partir do cloreto de chumbo $\left(\mathrm{PbCl}_{2}\right)$. As soluções que simularam o efluente continham concentrações de 1, 2, 5, 10, 20 e $50 \mathrm{mg} / \mathrm{L}$.

Por fim, as isotermas de adsorção foram obtidas pelo método do banho finito baseado no balanço de massa do adsorbato. Massas conhecidas e iguais do adsorvente foram postas em contato com volumes constantes e iguais de soluções de chumbo de concentrações diferentes. Os ensaios de adsorção foram feitos nas temperaturas de 30,50 e $65^{\circ} \mathrm{C}$. O equipamento utilizado foi o Banho Metabólico Dubnof a massa de adsorbato utilizada foi de $1,0 \mathrm{~g}$.

Após 6 horas de contato entre as fases, tempo suficiente para atingir o equilíbrio, a solução remanescente foi analisada por técnica de espectrofotometria. Por balanço de massa (Equação 1), calculou-se a massa de metal adsorvida no bagaço da cana-de-açúcar.

$$
q=\frac{V\left(C_{\text {inicial }}-C_{\text {final }}\right)}{m} \quad\left[\frac{\text { mg de metal }}{\text { g de bagaço }}\right]
$$

Em que $V$ é o volume da solução, $\mathrm{C}_{\text {inicial }}$ e $\mathrm{C}_{\text {final }}$ são, respectivamente, as concentrações inicial e final do metal na solução, e m é a massa de adsorvente. 
Os dados experimentais foram ajustados aos modelos de Langmuir (Equação 2) e Freundlich (Equação 3).

$$
\begin{aligned}
& q=\frac{q_{m} K_{L} C}{1+K_{L} C} \\
& q=K_{F} C^{1 / n}
\end{aligned}
$$

Sendo: $C$ a concentração da solução de efluente sintética, dada em $\mathrm{mg} / \mathrm{L} ; \mathrm{K}_{\mathrm{L}}$ a constante de adsorção, dada em L/mg; $\mathrm{q}_{\mathrm{m}}$ a capacidade máxima de adsorção, dada em $\mathrm{mg} / \mathrm{g} ; \mathrm{n}$ o parâmetro de ajuste do modelo, adimensional; $\mathrm{K}_{\mathrm{F}}$ a intensidade de adsorção descrita como $\left(\mathrm{mg} \mathrm{g}^{-1}\right)\left(\mathrm{L} \mathrm{mg}^{-1}\right)^{1 / \mathrm{n}}$.

Os parâmetros termodinâmicos também foram objeto de estudos, foram calculados a partir das equações abaixo:

$$
\begin{gathered}
\Delta G=-R T \ln K_{a d s} \\
\ln K=\frac{\Delta S}{R}-\frac{\Delta H}{R T} \\
\Delta S=\frac{\Delta H-\Delta G}{T}
\end{gathered}
$$

Sendo: $\mathrm{T}$ a temperatura, dada em $\mathrm{K} ; \mathrm{R}$ a constante universal dos gases, dada em $\mathrm{J} / \mathrm{molK}$; $\Delta \mathrm{G}$ a variação da energia livre de Gibbs, dada em $\mathrm{J} / \mathrm{mol} ; \Delta \mathrm{S}$ a variação da entropia, dada em $\mathrm{J} / \mathrm{molK} ; \Delta \mathrm{H}$ a variação da entalpia, dada em $\mathrm{J} / \mathrm{mol}$.

\section{RESULTADOS E DISCUSSÃO}

A Figura 1 (a, b, c) apresenta a isoterma de adsorção de $\mathrm{Pb}^{2+}$ nas diferentes temperaturas estudadas. Da análise desta figura, observa-se que adsorção é mais favorecida a temperatura de $30^{\circ} \mathrm{C}$ (Figura 1a), o que corrobora o caráter exotérmico desta técnica de separação.

Os dados experimentais foram ajustados pelo modelo de Langmuir como também é apresentado na Figura $1(\mathrm{a}, \mathrm{b}, \mathrm{c})$. Através da regressão não linear destes dados foram obtidos os parâmetros $\mathrm{q}_{\mathrm{m}}$ e $\mathrm{K}_{\mathrm{L}}$ do modelo de Langmuir, assim como também foram determinados os parâmetros $n$ e $K_{F}$ do modelo de Freundlich, embora este último não tenha se ajustado satisfatoriamente aos dados experimentais como mostra a Tabela 1. 


\section{Congresso Brasileiro de Engenharia Química em Iniciação Científica Unicamp - Campinas - SP 19 a 22 de julho de 2015}

Figura 1 a,b,c - Isotermas de adsorção nas temperaturas de $30^{\circ} \mathrm{C}(\mathrm{a}), 50^{\circ} \mathrm{C}$ (b) e $65^{\circ} \mathrm{C}$ (c). (Dados experimentais $\circ$; Modelo de Langmuir -)

(a)

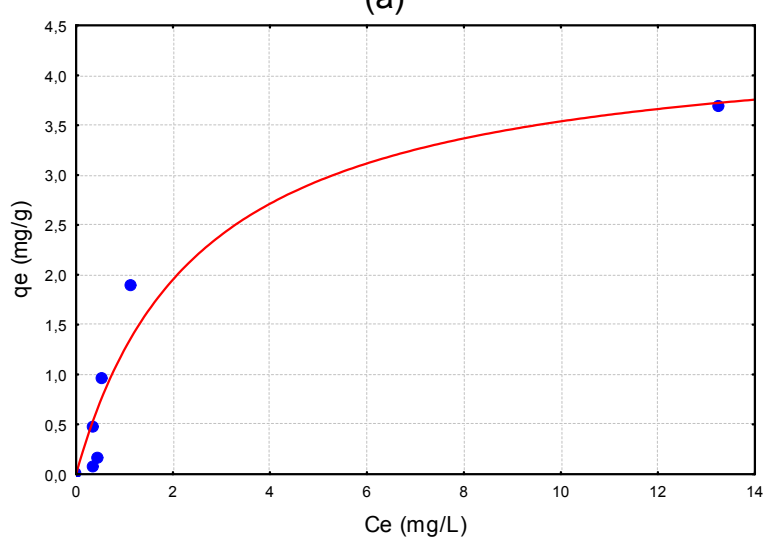

(b)

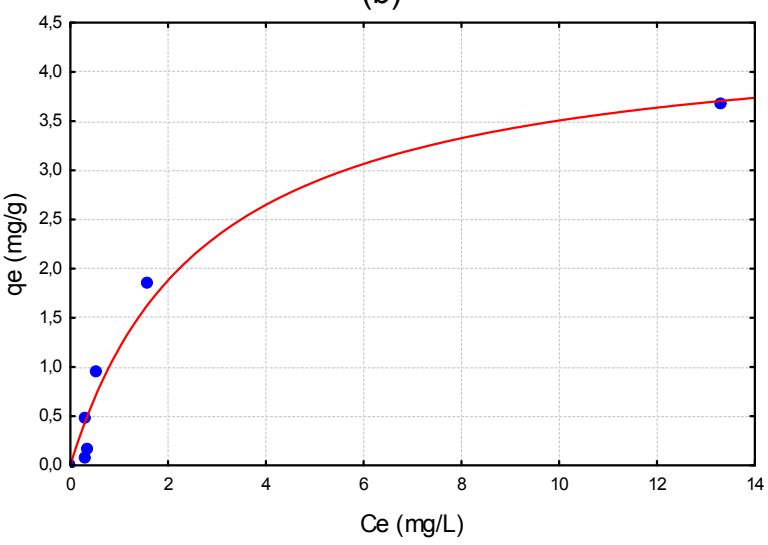

(c)

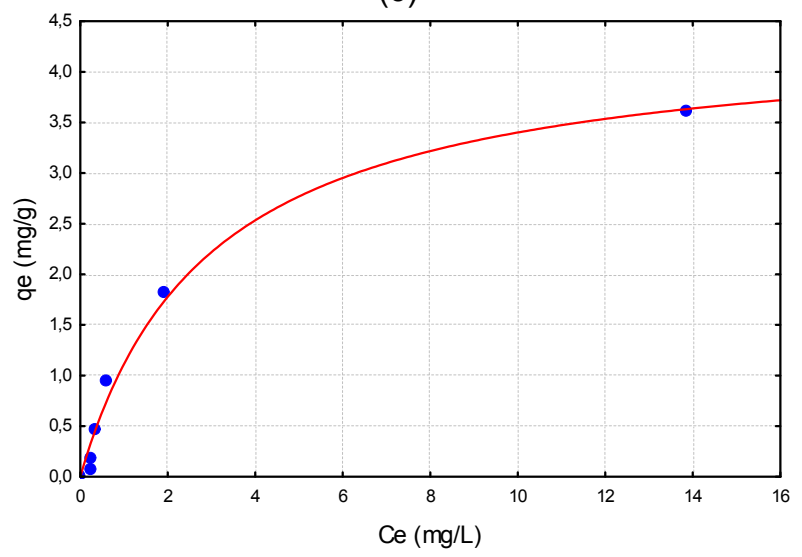

Tabela 1 - Parâmetros dos modelos de Langmuir e Freundlich

Modelo de Langmuir Modelo de Freundlich

\begin{tabular}{ccccccr}
\hline Parâmetros & $\mathrm{K}_{\mathrm{L}}\left(\mathrm{L} \mathrm{mg}^{-1}\right)$ & $\mathrm{q}_{\mathrm{m}}\left(\mathrm{mg} \mathrm{g}^{-1}\right)$ & $\mathrm{R}^{2}$ & $\mathrm{~K}_{\mathrm{F}}\left(\mathrm{mg} \mathrm{g}^{-1}\right)\left(\mathrm{L} \mathrm{mg}^{-1}\right)^{1 / \mathrm{n}}$ & $1 / \mathrm{n}$ & $\mathrm{R}^{2}$ \\
\hline $\mathbf{3 0}$ C & $0,39 \pm 0,16$ & $4,44 \pm 0,69$ & 0,925 & $1,01 \pm 0,28$ & 0,51 & 0,881 \\
\hline $\mathbf{5 0}$ C & $0,36 \pm 0,12$ & $4,47 \pm 0,53$ & 0,965 & $0,99 \pm 0,23$ & 0,51 & 0,923 \\
\hline $\mathbf{6 5}$ ㅇ & $0,34 \pm 0,08$ & $4,41 \pm 0,38$ & 0,986 & $0,96 \pm 0,19$ & 0,51 & 0,950
\end{tabular}

Quando o parâmetro 1/n (Tabela 1) do modelo de Freundlich apresenta valores entre 0,1 e 0,5 pode-se dizer que a adsorção é favorável (Samiey 2010), logo de acordo com os valores 


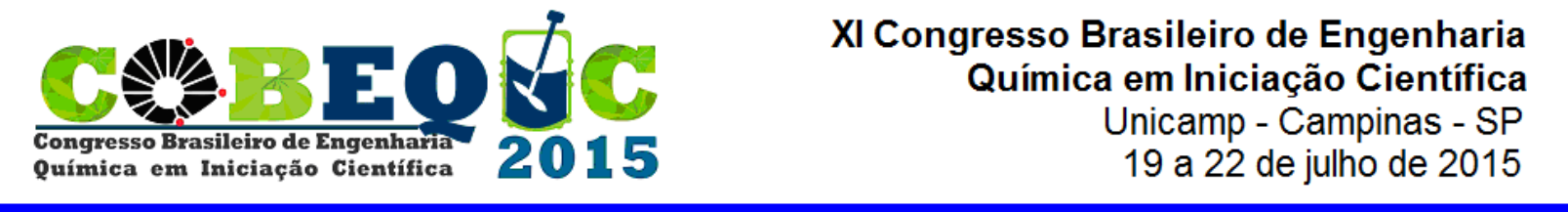

apresentados Tabela 1, $\left(\mathrm{R}^{2}\right)$ observa-se que o modelo que melhor descreve os dados experimentais é o de Langmuir.

Os parâmetros termodinâmicos, apresentados na Tabela 2, foram calculados com base na isoterma de Langmuir para as três temperaturas estudadas. A partir da Figura 2 (equação 5) foi calculada a variação de entalpia $(\Delta \mathrm{H})$. Utilizando a equação 4 calculou-se a energia livre de Gibbs $(\Delta \mathrm{G})$ e pela equação 6 determinou-se a variação de entropia $(\Delta \mathrm{S})$.

Figura 2 - Gráfico de Van’t Hoff para a adsorção de chumbo em bagaço modificado.

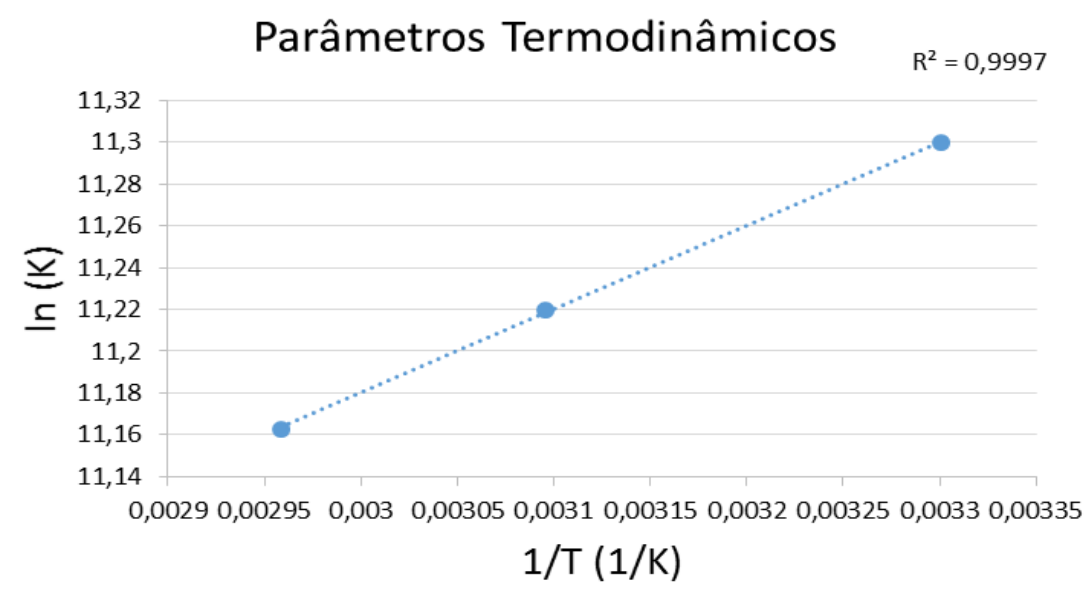

Tabela 2 - Parâmetros termodinâmicos

\begin{tabular}{|c|c|c|c|}
\hline $\begin{array}{c}\text { Parâmetros } \\
\text { Termodinâmico } \\
\text { s }\end{array}$ & $\Delta \mathbf{G}(\mathrm{KJ} / \mathrm{mol})$ & $\Delta \mathbf{H}(\mathrm{KJ} / \mathrm{mol})$ & $\Delta \mathrm{S}(\mathrm{KJ} / \mathrm{molK})$ \\
\hline $30^{\circ} \mathrm{C}$ & $-28,47$ & & \\
\hline $50^{\circ} \mathrm{C}$ & $-30,13$ & $-3,33$ & 0,083 \\
\hline $65^{\circ} \mathrm{C}$ & $-31,37$ & & \\
\hline
\end{tabular}

Os parâmetros termodinâmicos indicam que o processo é exotérmico e se enquadra como uma adsorção física pois o $\Delta \mathrm{H}$ está dentro da ordem de grandeza de $1-5 \mathrm{KJ} / \mathrm{mol}$ (Ruthven, 1984), o processo ocorre de forma espontânea segundo a energia livre de Gibbs deixando ainda mais claro que o é viável a utilização deste adsorvente para a remoção de chumbo de efluentes industriais, além disso o $\Delta \mathrm{G}$ também aponta para uma fisissorção de acordo com Liu (2010) o valor de energia livre de Gibbs para esse tipo de adsorção situa-se entre $-20 \mathrm{~kJ} / \mathrm{mol}$ e $0 \mathrm{~kJ} / \mathrm{mol}$ e que para quimissorção o valor varia entre $-400 \mathrm{~kJ} / \mathrm{mol}$ e -80 valor $\mathrm{kJ} / \mathrm{mol}$. 


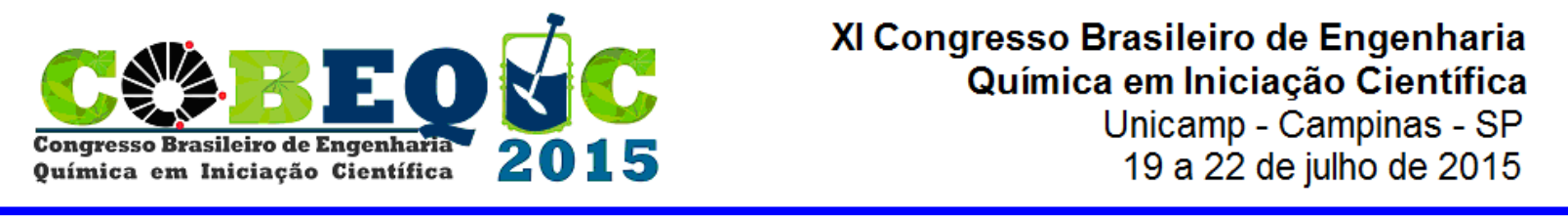

\section{CONCLUSÃO}

O bagaço de cana-de-açúcar quimicamente modificado apresentou-se como uma boa alternativa para a remoção de chumbo de efluentes industriais, destacando-se a adsorção a $30^{\circ} \mathrm{C}$. O modelo de Langmuir descreve melhor os dados de adsorção do sistema em estudo, sistema chumbo em bagaço de cana modificado, que o modelo de Freundlich.

Foi verificado que a adsorção realizada a $30^{\circ} \mathrm{C}$ apresentou melhores resultados, temperatura mais baixa entre as estudadas. Quanto aos parâmetros termodinâmicos o processo classifica-se como exotérmico e ocorre espontaneamente.

\section{AGRADECIMENTOS}

Ao Laboratório de Operações Unitárias da UFPB pela estrutura, ao Laboratório de Automação e Instrumentação em Química Analítica e Químiometria (LAQA) pelo fornecimento de reagentes e a Coteminas S.A. pelo apoio na realização das análises.

\section{REFERÊNCIAS}

BALANÇO ENERGÉTICO NACIONAL 2014: Ano base 2013. Rio de Janeiro: Empresa de Pesquisa Energética, 2014

LIU, Q.S., ZHENG, T., WANG, P., JIANG, J.P., LI, N., Adsorption isotherm, kinetic and mechanism studies of some substituted phenols on activated carbon fibers, Chem. Eng. J. 157 (2010) 348-356

SAMIEY, B., DARGAHI, M., Kinetics and thermodynamics of adsorption of Congored on cellulose, Central Eur. J. Chem. 8 (2010) 906-912.

SOUZA, R. S.; Santos. T. C.; Lima, L, R. Reuso do Bagaço da Cana-de-açúcar em Tratamento de Efluentes Industriais através da Adsorção. I Congresso Paraibano de Gestão do Lixo, Educação Ambiental e Sustentabilidade. Campina Grande - PB. 2009.

RUTHVEN, D. M. Principles of adsorption and adsorption Process. John Wiley \&Sons Inc. New York, 1984. 